\title{
ARTÍCULOS
}

\section{De la educación global a la biología del conocer. Comprender el sentido de la educación}

\author{
From global education to the biology of knowing. \\ Understanding the purpose of education
}

\author{
Dr. Sergio Toro Arévalo ${ }^{a}$, Dr. Jose M Pazos Couto, \\ Mag. Eivar Vargas Polanía ${ }^{c}$, Mag. Javier F. A. Vega Ramírez ${ }^{d}$ \\ ${ }^{a}$ Universidad Austral de Chile. \\ ${ }^{\mathrm{b}}$ Universidad de Vigo (España) \\ ${ }^{c}$ Universidad Surcolombiana, Colombia \\ ${ }^{\mathrm{d}}$ Universidad Austral de Chile. \\ sergio.toro@uach.cl \\ Telf.: (56) 63221262
}

\section{RESUMEN}

La educación es un proceso que se da de forma natural en el ser humano a lo largo de toda su vida, que se formaliza en un proceso educativo cuando sistematiza intenciones de terceros. Sin embargo, a lo largo de los últimos siglos, la estructura de la Educación ha variado muy poco, cuestión que hace necesario revisar efectivamente cuánto de los avances en Educación por parte de los grandes innovadores en Educación del S. XX ha sido recogido. Podemos advertir que prima actualmente un modelo economicista de la Educación y que la misma Evaluación educativa ha ido centrándose en esta perspectiva. Al analizar su situación actual vemos que también debe ser analizado el proceso de formación de profesores, para dar paso a un modelo que supere la simple dualidad cartesiana del "ser y conocer" hacia una propuesta coherente con la "biología del conocer".

Palabras clave: Educación, Evaluación, Biología del Conocer

\begin{abstract}
Education is a process developed in a natural way by human beings along their lives and which is shaped through an educational process when third person's intentions are systematized. However, along the last centuries, the education structure has varied too little requiring as necessary to check how much of the education advances made by the greatest innovators in education of the XX century have been gathered. We can warn that what primes today is an economic model of education and that the same educational evaluation has been centered through this perspective. When analyzing the current situation we observe that teachers' training process has to be analyzed to give path to a model that can overcome the simple Cartesian duality of "be and know" to a coherent proposal of "biology of knowing".
\end{abstract}

Key words: Education, Assesment, Biology of Knowing 


\section{INTRODUCCIÓN}

Hablar de educación se presenta como una tarea amplia y compleja, ya sea formal, no formal o informal. Al dialogar sobre ésta hacemos referencia de forma inherente al aprendizaje de las personas. La perspectiva varía en función de si la persona aprende "con o sin" la intencionalidad de un tercero, que pretende provocar aprendizajes en ella, o de si éste es intrínseco o extrínseco a la situación dada.

En la actualidad vivimos una realidad subjetiva que pretende cuantificar y medir todo lo referente a la educación, y en la que sin embargo, se identifica a los mejores docentes por lo que los diferencia con respecto a los otros. Destacando principalmente los que logran que los alumnos quieran aprender y disfruten haciéndolo (Jarauta y Medina, 2012). Asimismo, se tiende a estandarizar las políticas educativas, que se representan a nivel internacional en rankings educativos por países (PISA, etc.) y eso provoca comparaciones en virtud de unos estándares considerados como "los buenos" y que marcan tendencia en lo referente a la situación de un país y su nivel educativo (Saura y Luengo-Navas, 2015), lo que pretende indicar la competencia y la competitividad de los mismos.

Los sistemas educativos de los países de occidente contemplan la educación en diferentes fases: hasta los 6 años se entiende como educación global, a partir esta edad se parcializan los contenidos y los saberes, considerándose innovador quien trabaja de forma interdisciplinar, poniendo en cuestión el impacto real de la formación propuesta y su valor en la realidad diaria (Summo, Voisin y Téllez-Méndez, 2016). Y ya a partir de la educación secundaria se tiende a la especialización, y a orientar a las personas hacia el mundo laboral.

A pesar que todos los currículos y bases curriculares hablan de autonomía, de capacidad de aprendizaje, de competencias, la realidad nos dice que parece que "lo que hay que hacer con el conocimiento que se tiene a mano es introducirlo en las mentes de los alumnos y conseguir que se quede ahî" (Perkins, en Swartz, Costa, Beyer, Reagan y Kallick, 2013), como si se tratase del disco duro de un ordenador. Y los discursos de Decroly, de conocer a la persona para educarla mejor; de Freire, de huir de la educación bancaria hacia la conciencia crítica; la individualidad diferenciada de Piaget o Claparede; o el constructivismo de Ausubel, Bruner o Vygotsky, se transforman en métodos innovadores a pesar de pensar en otra educación desde principios del siglo XX.

Parece que en el currículum existe un doble discurso, el de los contenidos y el de las competencias, el de lo que debe ser la educación y lo que debe ocurrir en el aula. El maestro llega al aula y reproduce lo que han hecho con él en su experiencia de vida. Y el que se atreve a algo diferente, se arriesga a fracasar o a la excelencia, se expone a ser juzgado por la comunidad educativa. Pero ese riesgo se convierte a la vez en su principal aliado hacia el éxito, que sin lugar a dudas llegará si persiste con ilusión y voluntad de "buen hacer", pues hay un elemento en el proceso educativo que va más allá de la educación, que es la intencionalidad del acto educativo propuesto por el docente que por sí solo transmite una forma de ver el mundo y de vivir en él.

Mientras en educación se entienda este tipo de métodos como algo excepcional y no se los incluya en la forma de entenderla, mientras las emociones no formen parte del acto educativo, y el enseñar a pensar y cuestionarse el mundo no se convierta en algo habitual en la escuela, no se habrá producido una verdadera transformación de la misma, y búsquedas como el proyecto Spectrum (Cambridge, 1984) serán solo eso, un simple proyecto, y los 
siete saberes necesarios de la educación del futuro (Morin, 1999) solo serán eso, saberes necesarios de un futuro incierto que nunca llegará.

Existe una contradicción entre lo que las aportaciones científicas nos dicen sobre la relación entre las emociones, y el pensamiento como base de la actividad humana, con los aspectos cognitivos en los que tradicionalmente se ha centrado la escuela (Peña y Canga, 2009), dejando a un lado el resto de dimensiones del ser humano.

Entendemos el ser humano como un ser en el mundo que siente, se relaciona, piensa e interacciona con el mundo, un ser que se manifiesta a través de su motricidad, y la educación debe atender todas las dimensiones de la persona, pudiendo variar en diferentes momentos las dimensiones que potencia, pero sin aislar ninguna, pues el atender a éstas dota de sentido la propia acción educativa, y ayudará a todos los participantes del proceso a entender la complejidad de la realidad en que vive.

El ser humano, desde que nace, es un ser autónomo e inteligente, que se humaniza en la medida en que evoluciona su desarrollo, "el argumento fundamental es que a lo largo de la filogénesis y de la ontogénesis, el sistema nervioso ha integrado e incorporará relaciones, o conexiones, entre el movimiento, la percepción y el medio ambiente" (Avilés et al., 2014: 731).

Es obvio que la educación se caracteriza por una dimensión instructiva orientada hacia el aprendizaje intencionado de diversos contenidos, habilidades y actitudes, definidos desde un proyecto social y político expresado en el currículo formal, impregnando por lo tanto de una ética acordada explícita, del común o gran parte del grupo social comprometido en el esfuerzo de educar y ser educado (Toro, 2014). Al mismo tiempo, la educación por definición implica la libre adhesión de la propia persona o de quienes son responsables por ella, de lo contrario se trataría de otro proceso, más cercano al adiestramiento, domesticación o manipulación. El aprendizaje y su más cercano instrumento, el entrenamiento, pueden ser considerados elementos constitutivos del proceso educativo, pero en ningún caso lo agotarían (Maturana, 2004), pues su sentido más profundo sería, a nuestro entender, la comprensión, el empoderamiento y re-creación del trasfondo de la existencia y sentido de la sociedad y de sus proyecciones dentro del espacio-tiempo que tienen lugar. De manera que al educarse, se construyen sentidos y actualiza el modelo de relación social del que se forma parte dinamizando los modos de existencia del colectivo desde la actuación y operatoria autónoma.

Los seres humanos somos seres vivos que como tales, somos seres autopoiéticos moleculares, es decir, "una totalidad dinámica contenida en sí misma que, a través de su dinámica interna, define y realiza sus bordes como unidad discreta que existe sostenida espontáneamente por el entrelazamiento de la agitación molecular térmica y la configuración estructural plástica de las moléculas que lo componen" (Maturana y Dávila, 2015: 61). Esta definición desde la biología del conocer nos sitúa directamente en la constitución de seres vivos en primer lugar que se caracterizan por un operacional sensorial y relacional como dimensiones configuracionales de su vivir en una unidad ecológica individuo-nicho. De manera que las relaciones que se establecen desde la unidad ya mencionada lo son en tanto que bucle y no como epifenómeno. La consecuencia de tal aspecto es que lo conocido o el conocer tiene que ver sustantivamente con la organización y condiciones estructurales del ser vivo y su despliegue en acoplamiento estructural con su entorno, en función de la conservación de la existencia. Si el comportamiento que se despliega tiene acople con el entorno en los espacio-tiempos del devenir, podemos decir que ese comportamiento es 
inteligente, pues mantiene su condición organizacional y amplía sus repertorios operativos. Este aspecto en educación es muy importante dado que, si centramos la condición de inteligencia en los comportamientos desplegados tanto de estudiantes como docentes, podemos decir efectivamente qué es aquello que se aprende, más allá de los contenidos curriculares formales, pues en definitiva lo que se aprende son las relaciones vivenciadas, las formas de despliegue y en virtud del flujo emocional que las orienta, que da sentido y organización al co-devenir o convivencia de la escuela.

La disposición de cada ser vivo y de su conocer se encuentra en su emocionalidad que guía su operación. Sin embargo, dadas las características de eusocialidad de la especie humana (Wilson, 2012), ello solo tiene lugar en la conversación o diálogo con otros de su especie, es decir lo constitutivo de lo humano se da en la coordinación con otros humanos en la resonancia emocional que permite la relación, que al mismo tiempo es acto y significado. En ese devenir se constituye la individualidad y sobre todo el agente o actor:

la emergencia del sentido común, de la configuración autónoma de una postura adecuada que ha sido establecida por la historia de vida del agente...la clave de la autonomía es que un sistema viviente transcurre hasta el momento siguiente mediante una acción efectiva que surge de sus propios recursos. Y estos quiebres bisagras que articulan los micro mundos, están a la base del carácter autónomo y creativo de la cognición de los seres vivos (Varela, 1992: 11).

El conocimiento más natural e irrenunciable a cualquier ser vivo, y en especial lo humano, se genera desde este devenir de la experiencia en su entorno y es lo que nos permite establecer el principio de la autonomía, la distinción fundamental de todas. En este devenir de las experiencias con otros seres humanos de acuerdo a sus propias historias, se va construyendo lo que definimos como lenguaje y cultura.

Por tanto, donde sea que encontremos regularidades tales como leyes o roles sociales y consideremos que son dados externamente, habremos entonces sucumbido a la falacia de atribuir identidad sustancial a aquello que en verdad es una propiedad emergente de un proceso complejo, distribuido, mediado de interacciones sociales (Varela, 1992: 62).

"El estímulo sensorial que el organismo recibe depende directamente de la acción desplegada y de cómo el organismo se desplaza depende directamente de las consecuencias sensoriales de su acción previa" (Thompson, 2007: 368). En tal sentido, nos encontramos en un flujo operativo o enacción permanente, que se vuelca sobre sí misma para dar cada vez configuración al y acoplamiento con el entorno, siempre recursiva, siempre relacional. Como se muestra en la Figura 1, una experiencia se genera en la confluencia de todos los elementos y sistemas que configuran lo humano y su entorno inmediato. De manera que no podemos obviar o relativizar ninguno de ellos; al contrario, lo fisiológico se ve impactando e impactado por lo simbólico, y viceversa.

La acción como núcleo de la existencia desemboca en la experiencia, es decir el remanente particular que emerge y permanece desde el despliegue, post-acción. En palabras de Agamben, la experiencia "es ex-per-ientia, un provenir-de y un ir-a-través-de" (2015: 40). La experiencia nos permite ir decantando que lo que, enactuado, se simboliza y significa desde lo personal a lo cultural y viceversa, dependiendo de cada acción, su intencionalidad 
Figura 1: Ciclo de la acción, basado en Modelo del Arco Intencional de Freeman (1999)

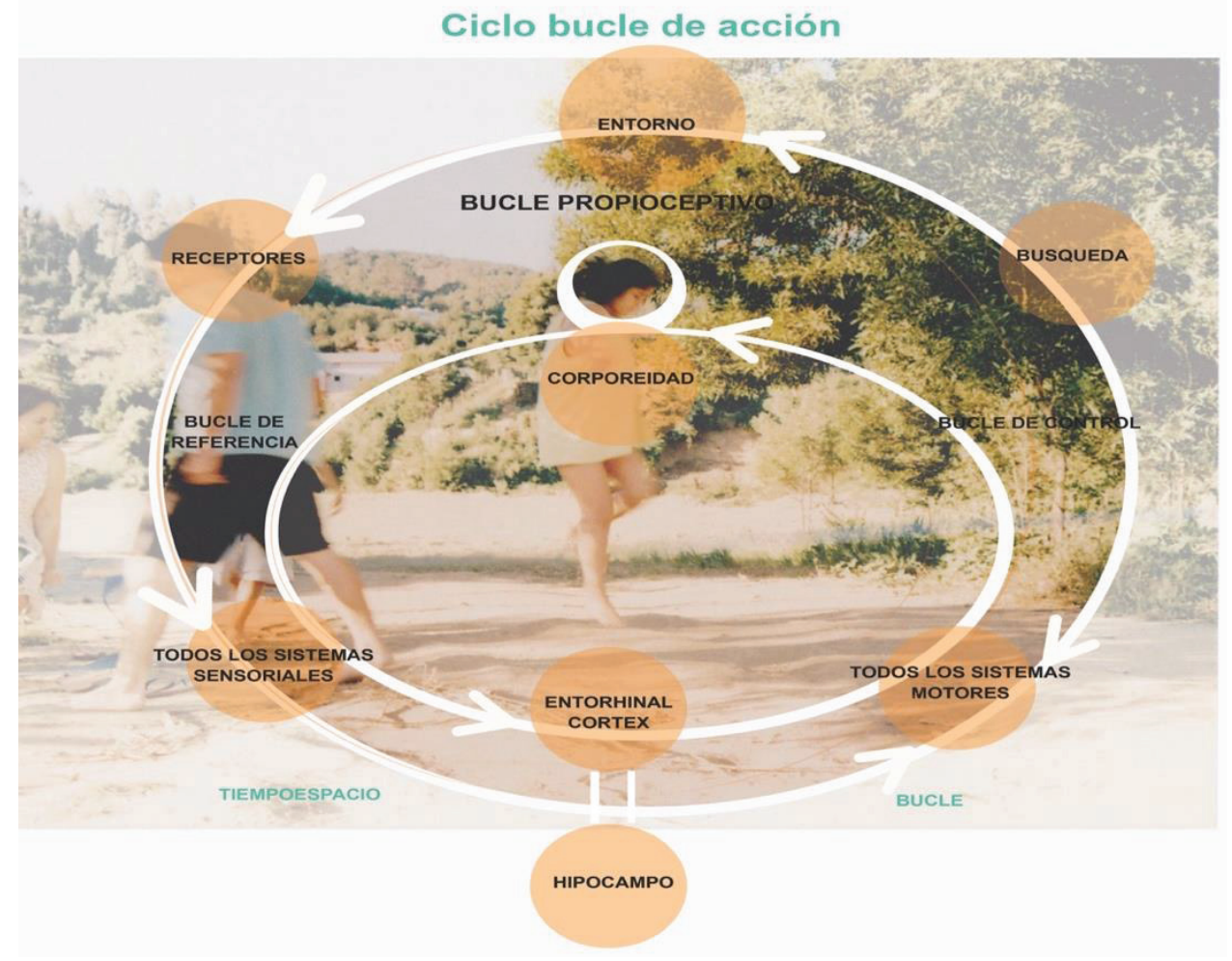

y sus respectivas contingencias. En el trascurso de cada acción, la configuración de los sentidos empleados, el contexto y sus condicionantes histórico-culturales, las densidades relacionales producidas, las emociones vividas en el transcurso, permiten la emergencia de la experiencia. Lo importante sin duda, es que esta experiencia siempre tiene lugar, y su dimensionalidad o importancia está dada en la autonomía con la cual se emprende, pues desde la comprensión de la Biología del Conocer, los seres vivos no son instruccionables, es decir, no aprenden porque se les enseña, sino por su propia dinámica y procesos internos en relación con su entorno (Maturana y Varela, 2003). Por su despliegue emocional y su dinámica configuracional interna, desde fuera se presentan perturbaciones que pueden ser dimensionadas sólo desde la estructura y dinámica autónoma. No obstante, esa dinámica tiene lugar, y por consecuencia sentido, dentro de las relaciones históricas y presentes de la especie y el entorno en que se actúa. Es en la resonancia emocional, expresada en coacciones y en un co-devenir, que construimos lo que denominamos mundo y realidad en el lenguaje (Maturana, 1999; Maturana y Dávila, 2015).

En tal sentido las propuestas que se relatan dentro del presente artículo son una excelente oportunidad, desde lo descrito, para adentrarnos en la compresión de que una nueva experiencia educativa se genera desde una visión sistemática y compleja de la experiencia, y al mismo tiempo de una caracterización pertinente de lo educativo. 


\section{ALGUNOS PLANTEAMIENTOS EXISTENTES EN TORNO A LA EDUCACIÓN}

Como ya indicamos anteriormente, los planteamientos educativos varían en función del nivel educativo o de la edad del educando. Para los primeros años, encontramos ya en los siglos XIX y XX, en toda Europa, diferentes métodos y/o escuelas: desde las Infant Schools de Owen; la escuela maternal Francesa; la propuesta en España de Montesinos, en la que la Educación Física asume un rol primordial; el método de Fröbel (y los Kindergärten), en el que el niño es protagonista y actor principal de su educación, junto a su madre; aquel propuesto por Decroly, en el que la globalización del conocimiento es crucial; las ideas de Dewey en cuanto al rol de la educación para comprender la realidad en los diferentes contextos y dotar de autonomía a las personas; o las aportaciones italianas de María Montessori, basadas en las tendencias naturales del ser humano y en las características cambiantes de los niños y niñas a cada edad; las hermanas Agazzi, para las que la motricidad y el ambiente familiar adquieren un rol principal en la educación; o las escuelas de Modena, que integran la escuela a la ciudad (Sanchidrian \& Berrio, 2010). Todas estas propuestas siguen vigentes en la actualidad y parten de un núcleo común: el construir la educación en una experiencia de vida útil para los individuos y favorecer el progreso social a través del libre pensamiento.

Nos encontramos asimismo con propuestas como la escuela de Ginebra, cuyos principales representantes son Claparede, Piaget, Audemars y Lafendel, que se centraban en la educación en los primeros 10 años de vida de los niños y niñas, utilizando una metodología dinámica que respetaba la espontaneidad de los educandos. Otra propuesta muy en vigor en la actualidad es el constructivismo, que tiene como principales valedores a Vygotsky, Bruner y Ausubel, que entiende que el conocimiento es una construcción del ser humano, planteando que el maestro no es agente de transmisión de conocimiento, sino facilitador de la construcción de aprendizajes por parte de los niños (Pérez-López y Juanvera, cit. en Sanchidrian y Berrio, 2010).

La gran mayoría de las propuestas están pensadas para los primeros años de vida, donde paradójicamente la educación no es obligatoria en un gran número de países, aunque no por ello menos importante. Existen otras propuestas similares que pueden abarcar un rango mayor, como el proyecto Spectrum, desarrollado por Richard Feldman, de la Tufts University; y por Howard Gardner, del Proyecto Zero, de la Harvard University, que aplica la teoría de las inteligencias múltiples de Gardner (Varela y Plascencia, 2006).

En la actualidad, las instituciones públicas y privadas han tratado de definir las competencias clave para la educación en el siglo XXI, y se evidencia en estos modelos una justificación de tipo economicista, que prioriza y reduce el papel de la educación para el siglo XXI a la producción de la riqueza y a la empleabilidad (Esteve, Adel y Gisbert, 2013). Estos planteamientos nos ponen en un escenario neoliberal que se olvida de la encarnación del conocimiento y del crecimiento personal en busca de la autorrealización y la felicidad de las personas, y plantea un escenario de producción y más producción, donde los planteamientos pedagógicos buscan la eficiencia más que la búsqueda de una educación global en un escenario de libertad que plantee una pedagogía crítica (Freire, 2002).

En este sentido, se vuelca la responsabilidad educativa en la escuela, y este foco institucional "se acompaña a menudo de una excesiva preocupación por el rendimiento escolar, siendo planteado como meta casi única por políticas educativas y administraciones" (Parrilla, Muñoz-Cadavid y Sierra, 2013: 16), y es en este momento en el que se pierde la perspectiva de una educación global, del que la escuela es un elemento más del eje 
vertebrador que constituye la formación de las personas y su integración en la sociedad de la que forman parte.

Cada día acaparan más la atención los problemas al parecer poco resueltos, entre ellos cómo lograr el dominio o maestría en un campo de conocimiento, cómo desarrollar competencias o un aprendizaje complejo, cómo lograr la transferencia del conocimiento y convertirse en aprendices autodirectivos, cómo procede la construcción conjunta del conocimiento (Hernández Rojas y Díaz Barriga, 2013).

Hay otro autores, como Igelmo Zaldívar y Laudo Castillo (en prensa), que nos hablan de un paradigma pedagógico emergente, y que denominan como la pedagogía líquida que tiene que ver con escuelas libres o espacio escolares autogestionados, que se confrontan al planteamiento de una escuela neoliberal poco crítica, basada en la producción/ productividad, en evaluaciones de currículos y bases curriculares que parten de buenos principios, e incluso de buenos principios de evaluación, pero que se desvirtúan con las evaluaciones externas y comparativas que se dedican a establecer rankings de calidad, más que centrarse en la calidad interna y la verdadera utilidad de la educación en la que no tiene razón de ser hablar de fracaso escolar.

\section{EL SENTIDO DE LA EVALUACIÓN ACTUAL DE LA EDUCACIÓN Y DE LOS SISTEMAS EDUCATIVOS}

En la actualidad se habla de estándares de calidad en las instituciones educativas de cara a conseguir una educación de calidad. Para ello, la evaluación es una herramienta que debe permitir conocer la realidad de dichas instituciones de cara a conseguir una buena calidad educativa, y esto no se consigue sin la participación de todos los agentes involucrados en el proceso educativo (Vital Carrillo, 2016). Asimismo, esa evaluación se verifica posteriormente por agencias externas, que en teoría validan esas evaluaciones y la acreditan. Y es que parece que existe en la actualidad la necesidad de contar con instituciones educativas que sean punteras; parece que prima estar en los primeros lugares de los rankings, más allá de que sean índices de calidad relativos.

Se hacen rankings de universidades, de colegios, de profesores, y aunque no sepamos lo que miden o lo que valoran, parece que los que están en primera línea son los mejores, ¿Dónde está lo educativo de estos rankings? Acaso la vida, o la calidad de esta, ¿consiste en ser más o tener más que los otros? ¿la felicidad tiene que ver con el estatus social? ¿la educación pretende como finalidad última que seamos los mejores en nuestro entorno o en el mundo? Si esto es así, la educación y/o el sistema educativo habrá fracasado antes de empezar. Pues una cosa es que la escuela, la universidad, la educación alcancen unos mínimos necesarios de calidad que garanticen una buena educación, y otra diferente es que establezcamos rankings de cuáles son los mejores y cuáles los peores. Si hay un millón, solo uno será el bueno, y los demás serán perdedores, ¿qué modelo de educación pretende eso? Acaso si todos alcanzan los mínimos establecidos como estándares de calidad educativa ¿no son buenos centros? De ser así, esos mínimos de los que hablamos no estarían bien delimitados.

Debemos replantearnos cuál es el papel de la educación y a su vez el rol de la escuela, revisar tanto los objetivos que busca cumplir en la sociedad, como sus modos de organizarse y funcionar para plasmarlos (Giménez Giubbani, 2016). Parece asumido que la educación debe centrarse en el alumno, y que debe atender al contexto en que se lleva a cabo, y la 
finalidad sea el alcanzar el pleno desarrollo del ser humano. Ante esto nos preguntamos si tiene sentido el establecer rankings y la respuesta está clara: sí, si pretendemos una sociedad que marque diferencias cada vez mayores entre ricos y pobres; no, si buscamos una sociedad en la que primen los valores humanos y la igualdad de derechos.

El quid de la cuestión no está en el crecimiento económico, sino en la redistribución de ese crecimiento en la población, pues si se concentra en un grupo de privilegiados, socialmente estaremos retrocediendo, y siendo más pobres, creando una sociedad menos justa. En esto también tiene mucho que decir la educación.

Existen muchas variables que influyen directamente en estas percepciones, y una de ellas, muy destacable, es la económica. Si se incentiva a los centros escolares con recursos económicos en función del alumnado que atraen y de los resultados educativos, estamos perdiendo la esencia de lo que se pretende (educar) y estamos fomentando una competitividad absurda, que nada tiene que ver con la calidad del profesorado o de la educación, y que puede responder a otros intereses particulares (Rivas, 2015).

Si bien es necesario evaluar la calidad de la Educación y exigir un nivel mínimo que garantice la consecución de objetivos educativos en los centros de enseñanza, no es ético el incentivar a los "mejores" más que a los que cumplen con sus labores y objetivos, incluso ayudar en lo necesario a quien no cumple los objetivos para mejorar la calidad educativa. Pues hablar de educación, es hablar de futuro, es hablar de inversión en personas, en cultura, en valores sociales y en prosperidad social.

No se ha conseguido superar la enseñanza organizada en disciplinas, esto ha superado a los propósitos de formación de la escuela que nos hablan de competencias para la vida, y de la competencia de aprender a aprender. Como nos dice Morin, "los analfabetos del siglo XXI no serán los que no sepan leer ni escribir, sino los que no puedan aprender, desaprender y reaprender" (2011: 144). En esta línea, deberíamos establecer mecanismos de evaluación que valorasen estas capacidades en los educandos, y establecer acciones que favorezcan y estimulen que los docentes y los centros educativos se centren en formar personas competentes y preparadas para la vida actual en el contexto en que viven.

Otra de las consecuencias que surgen con las evaluaciones de los sistemas educativos es que hacen emerger conclusiones en función de los resultados, y pone en tela de juicio a todo lo que forma parte del sistema, como el profesorado, poniendo en cuestión en muchos casos la calidad de su formación o de su preparación como profesionales, analizando las consecuencias de dicha evaluación, y no el sentido de la misma.

\section{EL PERFIL DE LOS EDUCADORES Y SU FORMACIÓN PARA AFRONTAR LA EDUCACIÓN GLOBAL}

A la luz de este panorama vale la pena preguntarnos, ¿qué es la educación? ¿Qué queremos de ella? ¿Para qué educamos? ¿Qué tipo de hombre y mujer queremos educar? y ¿qué sociedad y país queremos? Estas preguntas deberían tratarse en cada contexto y escenario en donde se desarrolle la práctica educativa y, aún con mayor intensidad, en la escuela, de manera que sus respuestas sigan el camino de lo íntimo, lo apropiado, lo encarnado en la vivencia, en lo cotidiano, en lo popular, en lo comunitario, en lo que se es y lo que se desea ser. Tal vez por este camino podríamos hallar una escuela apropiada en lo situado, lo local y lo contextual. Una educación que camine desde la ética, la democracia y la criticidad, 
una escuela que le muestre a sus actores el profundo significado de la realidad con la cual se deben enfrentar.

Pero, por el contrario, nos encontramos con una escuela que nos arrastra desde el dualismo cartesiano a la fragmentación humana. Ha sido tal su efectividad, que nos han hecho sentir como un algo divisible: cuántas veces no nos hemos referido a nosotros mismos como razón y cuerpo, o mente y cuerpo, en sí, un ser que se puede entender por partes, en quien prima la razón como la única poseedora y capaz de crear el conocimiento, de distinguir lo bueno y lo malo, lo falso y lo verdadero, debido a que ella y solo ella es inteligible, inmune al contexto, a lo vivido, es ella y solo ella, quien puede otorgarle al ser la capacidad de abstraerse del mundo y de hacerlo poseedor de la verdad.

Ha sido la visión del ser desde el dualismo cartesiano uno de los elementos de la base constituyente sobre la cual se ha construido la educación y la escuela. Esto ha motivado que en ellas cueste el reconocimiento de la emoción, de lo vivido en el contexto y de lo aprendido antes de llegar a la escuela. La educación y la escuela desde la base cartesiana del ser y conocer, revelan un atentado contra la pertenencia social, cotidiana y académica, debido a que solo reconoce a la razón como forma abstracta de aprendizaje, negando la cognición en y desde el emocionar humano en despliegue de su acción, a partir de lo íntimo, lo apropiado, lo comunitario y lo popular, que dibuja su configuración como ser en su andar.

Objetivadas en su desarrollo hegemónico del modelo neoliberal, escuela y educación, rompen con las dinámicas de la sociedad, haciéndose tan anacrónicas que parecieran detenidas en tiempos pasados. Se debe aclarar que lo mencionado sobre la educación, la escuela y sus andares no corresponden a un desarrollo natural o espontáneo de las mismas, sino que se producen y reproducen de manera intencionada en la manipulación y dominación de la sociedad y de todas sus aristas como estrategia para naturalizar el modelo neoliberal, desde la educación bancaria (Freire, 2003:45).

Aquí entra un factor clave. El rol de los profesores. La profesión docente se ha planteado como nuclear y responsable de la formación de lo social y cultural de las generaciones futuras, por lo que éste ha sido caracterizado básicamente como un líder social (Dorfsman, 2015).

Nava-Amaya y Rueda Beltrán (2014), afirman que:

el argumento de evaluar a los maestros con base en los resultados de evaluación de sus alumnos se sustenta en el principio que establece que un buen docente es aquél que logra que sus alumnos aprendan. Esta lógica deja de lado la evidencia de que los resultados de los alumnos en las pruebas estandarizadas son producto de diversos factores, muchos de los cuales se encuentran fuera del control del docente y de la escuela (2014: 6).

Y además deja en manos de esos resultados de los alumnos el cuestionamiento de la formación de los docentes, sin cuestionar si las pruebas de evaluación están adaptadas en forma y contenido a la forma en que el alumnado aprende y en cómo manifiesta o es capaz de poner de manifiesto esos aprendizajes.

Y es que a veces nos olvidamos de aquella frase de Zubiri (2006), en la que afirmaba que la constitución del mundo humano es previa al encuentro con los otros y fundamento de este encuentro, y es en ese encuentro en el que se muestran actitudes, saberes, acciones e interacciones, también en ese encuentro se producen la mayoría de los aprendizajes y condicionamientos.

En la formación de los docentes existen al menos dos vertientes, la formación inicial y la permanente, pero antes de ellas está en planteamiento qué hacemos de la educación y de 
lo que debe perseguir. No se puede pretender una reforma en la educación sin una reforma en el pensamiento, y viceversa. Si pretendemos una educación global, debemos educar para ella, y formar a los docentes en ella, y eso implica un acto de valentía, pues todo lo que implique un cambio a nivel de pensamiento con enfoque en la educación supone un desafío al orden social establecido (Motta, 2000).

Por tanto, igual que debemos pensar qué educación queremos, qué tipo de persona pretendemos, también debemos plantearnos cuál será el perfil del docente que afrontará esa tarea y qué formación debe recibir. Las tendencias y escuelas educativas que citamos anteriormente (Dewey, Piaget, etc.) tenían claro el tipo de educación que buscaban y el rol del docente entre otras cuestiones.

En la actualidad, la docencia universitaria que se enmarca en el Espacio Europeo de Educación Superior (EEES) avanza en un modelo de aprendizaje constructivista, que procura un alumnado autónomo que autogestiona sus aprendizajes. Esta forma de entender la docencia en la formación docente debería implicar en un futuro próximo un cambio en la forma de plantear la docencia en niveles inferiores, aunque solo sea por la imitación del cómo han aprendido, de la imitación y del comprobar qué es factible. Por eso es de vital importancia que se perciba como efectiva y eficiente esta forma de plantear el proceso educativo.

Olmedo-Moreno plantea que

los modelos metodológicos docentes, llevan implícitos un tipo y una forma de docencia al igual que ésta implica un modo de evaluación. Existen múltiples clasificaciones de los modelos metodológicos en Educación Superior, aunque todos coinciden en establecer al menos dos tipos: tradicional o cuantitativo, basado en un aprendizaje cuantitativo, y el alternativo o cualitativo/constructivista, donde el aprendizaje se basa en la comprensión, significado y la construcción de un marco conceptual por el propio estudiante (2013: 413).

Dorfsman (2015) nos plantea cuatro dimensiones necesarias en la formación docente, que podemos ver en la siguiente figura:

Figura 2: Dimensiones necesarias en la formación docente (adaptado de Dorfsman, 2015)

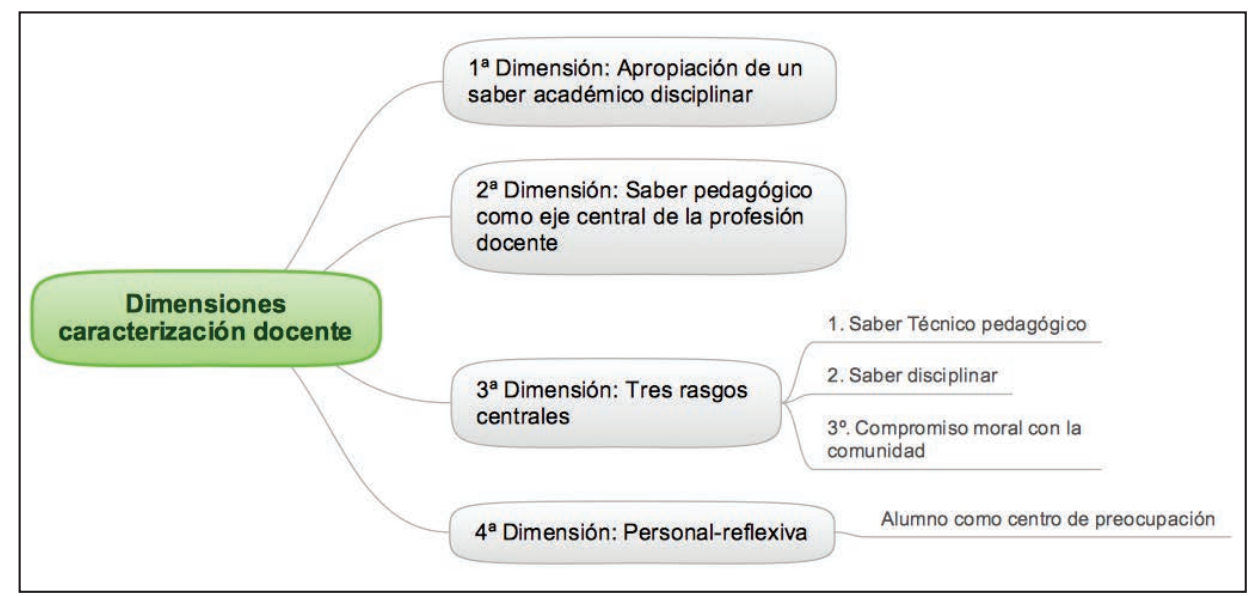




\section{LOS APRENDIZAJES EN LAS DISTINTAS ETAPAS DE LA VIDA}

Es necesario desentrañar la maraña en que habita la educación y la escuela, en quienes se escudriñan de manera silenciosa pero firme los objetivos neoliberales, que en su empeño de logro están dispuestos a devorar cualquier intención, deseo o acción que se resista a sus desarrollos. Como lo señala McLaren, "el conocimiento adquirido en la escuela nunca es neutral u objetivo sino que está estructurado en formas particulares; sus prioridades y exclusiones participan de una lógica silenciosa profundamente arraigada a los nexos de poder" (Mclaren, 2005: 105).

Pero son la misma educación y la escuela, en tanto que formas y escenarios de emancipación y de construcción de movimientos de resistencia las que podrán conducir a configurar caminos variopintos, que den lugar a formas diferentes de ser y estar en el mundo, formas diferentes y de resistencia a la forma neoliberal y cartesiana.

Se insiste en la necesidad de reconfiguración desde y en el ser, desde lo que somos en lo humano, pero ya no desde el dualismo cartesiano que nos impone el modelo de educación y escuela neoliberal, sino entendiendo al ser, en palabras de Varela, como

una trama de identidades sin centro que se encuentra en una interacción dinámica y fluida, en la cual cada aspecto que es enfrentado o desarrollado desde una identidad afecta a los demás, [...] un ser indivisible que no puede ser trabajado o desarrollado por partes sino que por el contrario cada parte actualiza el conjunto y en conjunto actualiza la parte (2000: 98).

Se trata, entonces, de entendernos como biología que conoce de manera permanente en y desde las emociones que jamás se agotan, y que mutan a cada paso. De esta forma, ya no podríamos hablar de una educación intelectual instaurada en la razón como centro y eje dinamizador educativo, sino de una educación desde un ser indivisible y en acto de trascendencia, en despliegue de sus acciones, intenciones y sentidos, es decir, un ser en cognición encarnada en todo lo que es, y la significación y configuración que ha realizado desde lo vivido en su relación dialogante con los otros y el mundo.

Varela nos invita a pensar en una forma de ser emergente, diferente a la impuesta por la educación global, que genera pasión por una forma también diversa del conocer, en una biología indivisible, que se implica de manera perenne en su historia y cultura, generando avatares que hacen que jamás seamos los mismos. Es la biología del conocer la forma como el ser se apropia y sitúa en su contexto y comunidad. En este sentido, nos referimos a una biología que se hace en la historicidad y caminar cultural del ser, y que supera la biología de la fiscalización.

Apropiarse de la biología del conocer sería una mudanza de la cognición unidimensional asentada en la razón, para situarnos en la cognición humana que se construye con lo que el ser es, lo que vive, lo que sueña, lo que lo provoca, lo apasiona y lo resiste. En este sentido, nos encontramos con un ser "que conoce, vive, siente, piensa, hace cosas, se desplaza, crece, trasciende, se emociona, se relaciona con otras personas y el mundo que le rodea" (Trigo, 2000:78), un ser histórico, que se ha configurado y existido solo desde su implicación mutua con y en la sociedad.

En la biología del conocer el ser se encuentra como actuante en el conocer permanente, pero ya no de manera aséptica y abstracta, sino implicado y encarnado en el devenir sobre el cual hemos sido a través de nuestra historicidad y acto de ser siempre en emoción. 
Sobre esto, Maturana diría que "el ser humano no da un paso en el mundo sin estar en la emoción", y esto nos lleva a comprender que la cognición, es decir el conocer humano, sólo ocurre en y desde la emoción" (2002: 57).

Pero ¿qué son las emociones? Maturana y Varela se refieren ante ellas "como disposiciones corporales dinámicas que definen los distintos dominios de acción en que nos movemos. Cuando cambiamos de emoción, cambiamos de dominio de acción" (2003: 83), y esto indica que jamás estamos sin emoción, y que la misma razón o mejor el acto de pensar está fundado en la emoción.

El hombre y mujer en acto, es decir en cognición desde la emoción, no puede ser entendido como humano sin estimar su implicación en y desde su dialéctica histórica en lo social. En este sentido para entender lo humano, se debe comprender que se hace humano siendo con otros, es allí donde lo humano toma sentido. Como lo señala Freire, de esta forma:

fue reinventándose a sí mismo, experimentando sufriendo la tensa relación entre lo que hereda y lo que recibe o adquiere del contexto social que lo crea y lo recrea, como el ser humano fue convirtiéndose en este ser que para ser tiene que estar siendo. Este ser histórico y cultural que no se puede explicar solamente por la biología o por la genética, ni tampoco solamente por su cultura, sino que se constituye socialmente transformándose en un cuerpo consciente (Freire, 1997: 62).

Por lo anterior, para comprender lo humano, es necesario conocer cómo se hace en lo humano, y que sólo sucede y es posible en relación dialogante con los otros en la magnitud de su devenir. Ante esto, Maturana declara que "la esencia, trascendencia y evolución del ser como humano, solo ha sido y es posible a través de la colaboración y no de la competencia" (1991: 71), pues ésta se enmarca en la negación de su reconocimiento como semejante, rompiendo con la configuración de lo humano y lo social.

Nos ocuparemos brevemente en mostrar que la competencia está destinada a la negación de la configuración humana y, con ello, a la negación de lo social. La motivación para detenernos en la competencia se debe a que ella es una de las bases de la educación que hoy impera y que ha convertido a la escuela en el templo de veneración y exaltación de la competencia como ser y estar de la práctica educativa, siendo ésta su principio, su fin y su único camino posible.

Esto indica que caminamos sobre una educación y escuela que niegan el reconocimiento del otro como ser legítimo y semejante, y que en su lugar tienen como objetivos la creación de seres competentes, seres orientados a la superación y el desprecio del otro, que buscan de manera voraz ser los mejores desde el combate y la anulación de los otros.

Para la escuela desarrollada desde la competencia no es necesaria la educación, solo le basta la instrucción, y es por eso que la usa como su forma de racionalización. De esta manera la escuela de hoy instruye desde la recopilación de cursos y contenidos que los hacen abstractos, anacrónicos, desposeídos de criticidad, comprensión histórica, reconocimiento de los problemas, de las significaciones humanas, de los fenómenos sociales y de la participación comunitaria y popular que se sitúan en los contextos, lo cual atenta contra la ética, la libertad y la democracia, haciéndolos inexistentes y dando paso a los procesos instructivos y de entrenamiento.

Las presencias humanas son diversas y coloridas. Es el ser y la vivencia de su motricidad, un lenguajear permanente en el mundo. De esta forma somos seres que en el 
lenguajear de la vida nos hacemos cultura, historia, política, sexualidad, espiritualidad y todo lo demás que como ser nos convoca.

La posibilidad de ser es infinita, es imposible de predecir, de destinar y sólo se conoce en el acto mismo de conocer, de estar con el otro en el andar del mundo. Y es este mismo andar el que nos hace multicolores y nos impregna de formas diversas de ser, sentir y estar.

La escuela es un espacio de encuentro de esas formas multicolores. Son esas formas las que se manifiestan y no el cuerpo como estado físico. No es el movimiento mecánico el que se encuentra, sino la motricidad en expansión y despliegue del ser en y desde sus emociones en un conocer permanente.

Sumado a lo anterior, una de las situaciones que ha lacerado a la educación global y tradicional es creerse el único espacio de configuración o de "educación" del ser, sin comprender que el conocer es un acto biológico que vive el ser humano, y que no distingue ni se reduce a espacios específicos, sino que sucede en cada instante, que surgen en el trecho de su vida y lo encarnan. Sería un profundo error, seguir creyendo que llegan a la escuela seres neutros, vacíos, listos para ser moldeados y manipulados, pues esto es irreal, debido a que "el hombre es conocido como la emanación de un medio social y cultural" (Le Breton, 2002: 142).

De esta forma, el ser se encuentra en despliegue con todo lo que lo posee, develando su forma de ser, sentir y lenguajear. Es también rostro, piel y cicatrices. Es también, al mismo tiempo, historia y cultura. Es la biología del conocer, la posibilidad de reconocer la configuración perenne del ser. Es allí donde el ser se hace y deshace, siendo jamás el mismo.

Es la escuela un encuentro de vibraciones de seres que se configuran, pero estas vibraciones han contado siempre con la intención de control de la educación global. Es esta misma acción de control parte de su historia y cultura, encargadas de enseñar, o más coherente con los actuales tiempos, de instruir y adoctrinar; y es en esa intención que se deben comprender los actuales procesos de educación.

Al parecer a la escuela le cuesta la sensibilidad por la diversidad de las formas de aprender, y lo que existe es una mirada despectiva, o tal vez ni siquiera una mirada, sino la ignorancia que manifiesta el rechazo de la diversidad por medio de una apología de la instrucción, el adoctrinamiento y el cuerpo objeto y mecánico.

La apología del cartesianismo que hace la educación global pone a la razón como la única posibilidad en la escuela, y oscurece la comprensión del ser como complejidad, que conoce desde el entramado que hace y quiere hacer en y desde su vida. Al parecer, para los docentes su función profesional tiene sentido en el abordaje del humano como cuerpo físico medible y cuantificable, y no para el ser humano fenomenal que constantemente se hace en el mundo.

Lo que sucede en la escuela es un desgarro de lo que hace al ser único, presente y no presente. Es el desgarro de su historia, su cultura, sus contextos, sus pasos en el mundo que le proporcionan sentido a su andar. Insistir en ver a los seres sólo como objeto de intervención biológica, anatómica y motora, es negar su configuración social, su naturaleza, es negar su propia biología, pues ésta también se hace en los pasos del ser por la vida.

Reconocer la educación y el aprendizaje como acto presente del ser humano, es reconocer también el ser en el mundo de la vida en tanto que ser único y situado. Es un ser que toma sentido en su contexto, en las relaciones de sentido que allí emanan y vibran, y son estas relaciones de sentido las que lo humanizan y lo potencian en un avatar constante. 
Es necesario que la escuela y los profesores asuman la educación del ser como un acto permanente, que sucede en cada momento de la vida, en el mundo en el cual se ha hecho el ser y en el mundo que éste configura cada día, reconociendo su andar, su historia y su cultura. Se trata de un reconocimiento que aportará a una práctica educativa que tenga como referencia los intereses, sentidos y significaciones de quienes se encuentran y comparten en la escuela.

Dejar atrás la postura cartesiana y caminar a la comprensión del ser desde la biología del conocer, que aprende y educa a través de su historia y cultura en una construcción mundana, es entender en los seres humanos "las hibridaciones y entramados mostrados con su forma de lectura de la realidad que viven, y que en ocasiones los invisibiliza y les niega sus espacios, de las cada vez más restringidas oportunidades que se les ofrecen" (Hurtado, 2011: 132).

En este sentido, se trata de abrir sus espacios para la potenciación del ser en su conocer, su aprendizaje y educación, pero no en condiciones de control u ocultamiento, como sucede en la actual educación global tradicional, sino que en tanto que responsabilidad de la educación que se construye teniendo como punto de partida el propio ser y todo lo que lo encarna.

En relación con lo anterior Toro y Valenzuela manifiestan que:
el educar o ser educados nos remite a la tensión primordial y local del mundo que habitamos y que somos. Si en un sentido clásico la educación se centraba en lo universal y trascendente, lo que deberíamos constatar es que dicho proceso no ocurre desde la desnaturalización de lo vivido y de la cotidianidad, de lo histórico y sus correspondientes cuestionamientos esenciales y locales. No se camina sin punto de partida, sin el propio andar por muy guiado y favorecido que sea, no es posible renunciar a la tensión inicial del expresar e impresionar de la vida (Toro y Valenzuela, 2014: 54).

Educar en la comprensión encarnada que hace el ser desde esta biología del conocer es comprender sus posibilidades, potencialidades, temores, sueños, deseos, emociones, cicatrices. Es dejar atrás el trasnochado cuerpo-objeto vacío, y desposeído, para asumir el sujeto vivencial que en despliegue se apropia de sentidos y significados. Esto favorece un acto educativo situado en el aprendizaje de la vida. En este sentido, se vivenciará la comprensión del ser en su esencia fenomenal de conocer el mundo en un acto educativo de emancipación, en donde la práctica educativa debe ser en sí misma una práctica libertaria.

La apropiación de la biología del conocer es reconocer al ser que aprende y se educa durante el caminar de su historia, vida y mundo. De esta forma, se asistirá a su expansión, pero ya no controlándola, sino potenciándola, explorándola, conociéndola. En este sentido, se exigirá a la educación y la escuela "abrirse a la motricidad hecha en la calle, el barrio, con los amigos, el padre, los abuelos, para así potenciarla en la escuela" (Hurtado, Jaramillo, Zuñiga y Montoya, 2005: 91).

Se trata de una necesidad de abandonar la prepotencia de la educación global, en su posición como posibilidad única capaz de educar, para comprender una educación que se apropie del ser que conoce, aprende y se educa durante la vida misma, lo cual trasciende los límites que durante años ha establecido la escuela y el aprendizaje de la educación global, en donde se ha hecho creer que no es posible aprender y educarse sin la presencia de profesores, contenidos y planes. 
Es pasar de la educación del cuerpo de los tradicionales manuales de la sicología, biología, fisiología, anatomía, que se han hecho hegemónicos, para entrar en la educación del ser que conoce en cada paso de su historia y su cultura en el mundo. Para esto es necesario, como dice Freire, entender que "la práctica educativa no debe limitarse a la lectura de la palabra, a la lectura del texto, sino que debería incluir la lectura del contexto, la lectura del mundo" (2011: 60).

\section{A MODO DE CONCLUSIÓN}

Desde hace más de dos siglos existe una gran preocupación por la educación, y por encontrar coherencia entre lo que acontece en el entorno escolar y la rutina diaria; por enseñar a vivir y a encontrar el sentido de la vida.

En la actualidad, se está demostrando la capacidad de aprendizaje de las personas, y poniendo en cuestión lo que se denominaba procesos de enseñanza-aprendizaje, para pasar a ser únicamente procesos de aprendizaje, el rol del centro escolar no ha variado mucho, pero sí el rol de los docentes. Así se entiende desde la gran mayoría de las teorías contemporáneas.

Otro concepto que ha evolucionado considerablemente es el de la evaluación, que si bien es considerada necesaria por todos los grandes pensadores de la educación, en todo proceso educativo, está es puesta en cuestión cuando se utiliza como medio comparativo con otros individuos, o con otros sistemas educativos, por violar el contexto en el que se desarrolla, y por contribuir a provocar mayores diferencias de estatus social.

Las evaluaciones son muy buenas de cara al control interno de las instituciones, pero, mal utilizadas o mal interpretadas, pueden convertirse en lo contrario a lo que pretenden, en contextos en los que se determinen las políticas educativas a aplicar.

La formación de los docentes de los diferentes niveles educativos debe ser global, y desde el principio debe utilizar metodologías activas de aprendizaje que supongan un aprendizaje y un ser capaz de provocar aprendizajes en los educandos.

\section{REFERENCIAS BIBLIOGRÁFICAS}

Avilés, C., Ruiz-Pérez, L., Navia, J., Rioja, N. y Sanz-Rivas, D. (2014). La pericia perceptivomotriz y la cognición en el deporte: Del enfoque ecológico y dinámico a la enacción. Anales de Psicología, vol. 30 (2), 725-737.

Dorfsman, M. (2015). La profesión docente en contextos de cambio: el docente global en la sociedad de la información. Revista de Educación a Distancia - Docencia Universitaria en la Sociedad del Conocimiento, vol. 6. Recuperado desde http://www.um.es/ead/reddusc/6

Esteve, F., Adel, J., \& Gisbert, M. (2013). El laberinto de las competencias clave y sus implicaciones en la educación del siglo XXI. Paper presented at the II Congreso Internacional Multidisciplinar de Investigación Educativa (CIMIE).

Freire, P. (2011). Política y educación. México: Siglo XXI.

. (2003). Pedagogía del oprimido. Madrid: Siglo Veitiuno de España Editores.

. (2002). Concientización: Teoría y práctica de una educación liberadora (12a ed. vol. 1).

Buenos Aires: Galerna- Búsqueda de Ayllu.

(1997). La Educación como Práctica de la Libertad. México: Siglo XXI. 
Giménez Giubbani, A. (2016). El papel de la gestión de centros educativos en un modelo de aprendizaje basado en competencias. Revista Páginas de Educación., vol. 9(1), 5-15.

Hernández Rojas, G. y Díaz Barriga, F. (2013). Una mirada psicoeducativa al aprendizaje: qué sabemos y hacia dónde vamos. Sinéctica, vol. 40, 1-19.

Hurtado, D. (2011). Entramados Jóvenes y configuración de significaciones de deseo. Popayán: Feriva.

Hurtado, D., Jaramillo, L., Zuñiga, C. y Montoya, J. (2005). Jóvenes e Imaginarios de la Educación Física. Popayán: Universidad del Cauca.

Igelmo Zaldívar, J. y Laudo Castillo, X. (en prensa). Las teorías de la desescolarización y su continuidad en la pedagogía liquida del siglo XXI. Educación XX1.

Jarauta, B., y Medina, J. L. (2012). Saberes docentes y enseñanza universitaria. Estudios sobre educación, 22, 179-198.

Le Breton, D. (2002). La sociología del cuerpo. Buenos Aires: Ediciones Nueva Visión.

Maturana, H. (2004). Del ser al hacer. Santiago: J.C. Sáez Editor. . (2002). Formación Humana y Capacitación. Santiago: Dolmen. (1999). Transformación en la convivencia. Santiago: Dolmen. (1991). El sentido de lo Humano. Santiago: Editorial Universitaria/ Hachette.

Maturana, H., \& Varela, F. (2003). De Máquinas y seres Vivos. Buenos Aires: Editorial Universitaria/ Lumen.

Maturana, H. y Dávila, X. (2015). El árbol del vivir. Santiago de Chile: Prv Editores.

McLaren, P. (2005). La vida en las escuelas. México: Siglo XXI.

Morin, E. (2011). La Vía para el futuro de la humanidad. Barcelona: Paidós.

Motta, R. (2000). Complejidad, educación y transdisciplinariedad. Signos Universitarios, vol. 20 (37), 69-92.

Nava-Amaya, M., y Rueda-Beltrán, M. (2014). La evaluación docente en la agenda pública. Revista electrónica de investigación educativa, vol. 16 (1), 1-11. Recuperado desde http://redie.uabc. $\mathrm{mx} / \mathrm{vol16no1/contenido-nava-rueda.html}$

Olmedo Moreno, Eva M. (2013). Enfoques de aprendizaje de los estudiantes y metodología docente: Evolución hacia el nuevo sistema de formación e interacción propuesta en el EEES. Revista de Investigación Educativa, vol. 31 (2), 411-429.

Parrilla, A., Muñoz-Cadavid, M., y Sierra, S. (2013). Proyectos educativos con vocación comunitaria. Revista de Investigación en Educación, vol. 11 (3), 15-31.

Peña, A. y Canga, M. (2009). La educación emocional en el contexto escolar con alumnado de distintos países. European Journal of education and Psychology, vol. 2 (3), 199-210.

Rivas, A. (2015). América Latina después de PISA: lecciones aprendidas de la educación en siete países 2000-2015. Buenos Aires: Fundación CIPPEC.

Sanchidrian, C. y Berrio, J. (Coords.) (2010). Historia y Perspectiva actual de la educación infantil. Barcelona: Grao.

Saura, G. y Luengo-Navas, J. (2015). Biopolítica y educación. Medición, estandarización, regulación poblacional. Teoría de la Educación, Revista interuniversitaria, vol. 27 (2), 115-135.

Summo, V., Voisin, S. y Téllez-Méndez, B. (2016). Creatividad: eje de la educación del siglo XXI. Revista iberoamericana de Educación Superior (RIES), vol. 7 (18), 83-98. Recuperado desde https://ries.universia.net/article/view/1126/creatividad-eje-educacion-siglo-xxi

Swartz, R., Costa, A. L., Beyer, B. K., Reagan, R. y Kallick, B. (2013). El aprendizaje basado en el pensamiento. Como desarrollar en los alumnos los pensamientos del Siglo XXI. EU: SM.

Toro, S. (2014). La investigación del aprendizaje auténtico para y desde el aula. En Toro, S. y Vega, J. Investigación en y para la escuela. Valdivia: Ediciones Kultrún.

Toro, S., \& Valenzuela, P. (2014). Educación para y desde la Militancia y la transformación social: Nuevas racionalidades Didácticas. En A. Moreno, \& M. Arancibia, Educación y Transformación Social construyendo una ciudadanía Crítica (pp. 54-66). Valparaíso: Ediciones Universitarias de Valparaíso. 
Trigo, E. (2000). Fundamentos de la Motricidad. Barcelona: Gymnos.

Varela, F. (2000). El Fenómeno de la Vida. Santiago de Chile: Dolmen.

Varela, C. y Plascencia, I. (2006). El Proyecto Spectrum: aplicación y actividades de aprendizaje de ciencias en el primer ciclo de la Educación Primaria. Revista de Educación, vol. 339, 947-958.

Vital Carrillo, M. (2016). La evaluación externa y autoevaluación de instituciones educativas. Vida Científica. Boletín científico de la Preparatoria $n^{\circ} 4$, vol. 4 (7). Recuperado desde https:// repository.uaeh.edu.mx/revistas/index.php/prepa4/article/view/1996/2001

Wilson, E. (2012). La conquista social de la tierra. Barcelona: Debate.

Zubiri, X. (2006). Tres dimensiones del ser humano: Individual, social, histórica. Madrid: Alianza. 
